\title{
Thrombotic disorders (part 2)
}

N A Alli, MB BCh, FCPath (SA) Haem; J Vaughan, MB BCh, MMed (Haem), FCPath (SA) Haem; S Louw, MB BCh, MMed (Haem), FCPath (SA) Haem; E Schapkaitz, MB BCh, MMed (Haem), FCPath (SA) Haem; B Jacobson, MB BCh, MMed (Haem), FCPath (SA) Haem, PhD

Department of Molecular Medicine and Haematology, Faculty of Health Sciences, University of the Witwatersrand, Johannesburg; and National Health Laboratory Service, Johannesburg, South Africa

Corresponding author: N A Alli (nazeer.alli@nhls.ac.za)

Thromboembolic conditions are a leading cause of mortality, estimated to account for 1 in 4 deaths worldwide in 2010. Over time, the incidence and mortality rates of these conditions have declined in developed countries, but are increasing in developing countries. A delicate balance exists between procoagulant and anticoagulant factors within the vascular system. Numerous acquired or inherited conditions may tip the balance either way, i.e. towards a prothrombotic or prohaemorrhagic state. Acquired thrombotic disorders are the subject of discussion in this issue, the second of a 2-part series on thrombophilia.

S Afr Med J 2020;110(3):181-187. https://doi.org/10.7196/SAMJ.2020.v110i3.14634

Thrombotic disorders may be inherited, as discussed in part 1 (previous issue), ${ }^{[1]}$ or acquired. They are the subject of discussion in this issue, the second of a 2-part series in CME.

The process of coagulation involves activation, adhesion and aggregation of platelets to damaged endothelium, followed by deposition and maturation of fibrin, and begins almost immediately after injury to the endothelial lining of the blood vessel. Exposure of blood to the subendothelial area initiates two processes, viz. changes in platelets, and the exposure of subendothelial tissue factor (TF) to plasma factor VII (FVII). Platelets immediately form a plug at the site of injury, which is referred to as primary haemostasis. Secondary haemostasis occurs simultaneously where additional coagulation factors beyond FVII respond in a cascade to form fibrin strands, which confer stability to the platelet plug (Fig. 1). ${ }^{[2]}$

The TF-FVIIa complex is a crucial trigger for haemostatic responses in vivo, and excessive initiation of coagulation through this pathway can lead to thrombosis or consumptive coagulopathy. Increased TF-FVIIa complex formation can result from loss of vascular wall integrity and increased TF expression. For example, atherosclerotic plaques contain significant levels of TF, generally associated with monocytes/foam cells and smoothmuscle cells. ${ }^{[2]}$ TF expression can also be increased with malignancy, potentially leading to cancer-associated thrombosis. ${ }^{[3]}$ During sepsis, TF is expressed on monocytes, but is also expressed by endothelial cells in some areas, such as the splenic microvasculature. ${ }^{[4]}$
Causes of acquired thrombophilia are listed in Table 1.

A more detailed discussion is given below regarding acquired risk factors for thrombosis, as well as information pertaining to clinical entities characterised by a marked predilection to thrombosis (i.e. the antiphospholipid syndrome, heparininduced thrombocytopenia and paroxysmal nocturnal haemoglobinuria). Thrombophilia associated with specific clinical scenarios mainly affecting the arterial circulation and microvasculature (e.g. myocardial infarction, diabetes, hypertension, thrombotic

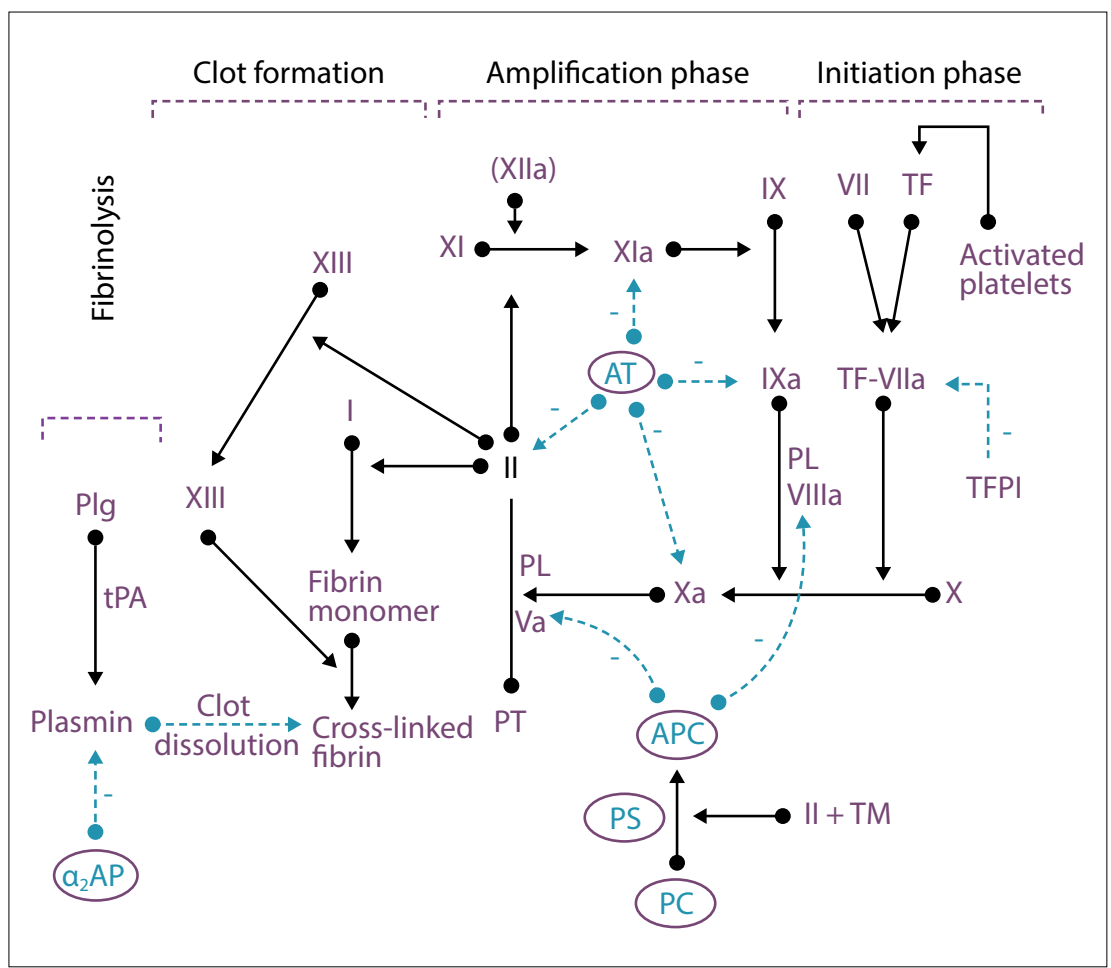

Fig. 1. Coagulation cascade. Dotted arrows indicate actions of natural anticoagulants and fibrinolytic factors. $(V, V I I I, I X, X, X I, X I I I=$ procoagulant factors; suffix $a=$ activated factor; $A T=$ antithrombin; $T F=$ tissue factor $I=$ fibrinogen $I I=$ thrombin; $P l g=$ plasminogen; $P L=$ phospholipid (on platelet membrane); TFPI = tissue factor pathway inhibitor; $t P A=$ tissue plasminogen activator; $P T=$ prothrombin; $A P C=$ activated protein $C ; P S=$ protein $S ; T M=$ thrombomodulin; $\alpha 2 A P=\alpha 2$-antiplasmin; $P C=$ protein $C$.) 
Table 1. Precipitating factors/conditions associated with thrombosis

Antiphospholipid antibodies

Atrial fibrillation/congestive cardiac failure

Chronic inflammation, e.g. TB, HIV, inflammatory bowel disease

Immobilisation/postoperative state

Malignancy/myeloproliferative disorders

Heparin-induced thrombocytopenia

Oral contraceptive use/pregnancy/oestrogen therapy

Diabetes mellitus/obesity

Hyperviscosity

Chronic haemolysis, e.g. SCD, PNH

Iatrogenic, e.g. HIT, indwelling venous catheter

Renal disease

TTP

Trauma

$\mathrm{TB}=$ tuberculosis; $\mathrm{SCD}=$ sickle cell disease; $\mathrm{PNH}=$ paroxysmal nocturnal haemoglobinuria $\mathrm{HIT}=$ heparin -induced thrombocytopenia; TTP $=$ thrombotic thrombocytopen purpura.

thrombocytopenic purpura (TTP)) are influenced by a multitude of factors and varied aetiologies. Such scenarios merit discussion on discipline-specific platforms and therefore do not fall within the scope of this CME. The scope of this discussion is limited to clinical entities associated with a marked increase in the risk of thrombosis. We conclude with a guide to the diagnosis and further investigation of suspected thromboembolic disease.

\section{Acquired risk factors for thrombosis Mycobacterium tuberculosis infection}

The risk of venous thromboembolism (VTE) is 1.5 times higher in patients with Mycobacterium tuberculosis (TB) infection than in uninfected individuals and relates to immobilisation, increase in procoagulant acute-phase reactants such as fibrinogen, decrease in the natural anticoagulants including protein $\mathrm{C}$ (PC) and protein S (PS), and mechanical compression of veins from enlarged lymph nodes. ${ }^{[5]}$ Active TB infection is also frequently linked to underlying infection with HIV, which further increases the risk of VTE. ${ }^{[6]}$ Concomitant HIV infection and VTE during TB treatment can result in clinically significant drug-drug interactions, as warfarin is metabolised by the cytochrome P450 (CYP450) pathway in the liver. Therapeutic agents that induce this pathway (e.g. rifampicin and nevirapine) or inhibit it (e.g. isoniazid and efavirenz), may result in under- or over-anticoagulation, necessitating more intense monitoring and management of anticoagulation therapy.

\section{HIV infection}

The incidence of arterial and venous thrombosis in HIV-infected patients is increased compared with healthy controls. The pathogenesis of the hypercoagulability in people infected with HIV is multifactorial and relates to an increase in procoagulant acute-phase reactants (Von Willebrand factor, fibrinogen and FVIII), a decrease in the level of natural anticoagulants (PC and PS), production of lupus anticoagulant antibodies due to immune dysregulation and endothelial dysfunction. Concomitant disease processes in HIV-infected patients, including secondary malignancies, additional infections, concurrent immobilisation and drugs, can also contribute to the increased thrombotic risk that has been recognised in VTE treatment and prophylaxis guidelines. ${ }^{[7-9]}$

\section{Renal disease}

Chronic kidney disease (CKD) is an established risk factor for arterial and venous thrombosis. The latter occurs 5.5 times more frequently in patients with CKD than in people with normal renal function. ${ }^{[10]}$ The hypercoagulable state in patients with CKD relates to procoagulant factors (antiphospholipid antibodies, artificial shunts and indwelling catheters), concomitant use of erythropoietin-stimulating agents, decline in fibrinolytic activity and decreased natural anticoagulant levels. Anticoagulation therapy in patients with renal dysfunction can be challenging owing to altered drug pharmacokinetics, necessitating dose adjustment and more intense monitoring. ${ }^{[10,11]}$

\section{Inflammatory bowel disease}

Patients with inflammatory bowel disease (IBD), the major forms of which are ulcerative colitis (UC) and Crohn's disease (CD), have a 3-fold increased risk of developing venous and, to a lesser extent, arterial thrombosis. The hypercoagulability in patients with IBD is multifactorial and not completely understood, but relates to chronic inflammation with increases in acute-phase reactants and cytokines, hospitalisation, surgery and indwelling catheters. VTE episodes usually correlate with disease activity, although these may occur during periods of remission. There is evidence that patients with IBD experience the first episode of VTE at a younger age than the general population, with an increased risk of recurrence, and that UC and CD pose an equal thrombotic risk. ${ }^{[12,13]}$

\section{Pregnancy/oral contraceptive use}

Among women, additional risk factors for VTE include combined oral contraceptives (OCs), hormone replacement therapy, pregnancy and the postpartum period.

Oestrogen use increases the risk of VTE as a class effect, which is dose dependent. The risk of VTE depends on the route of administration. There is lower associated risk with transdermal and intrauterine hormonal therapy, as well as with progesterone-only OC use. ${ }^{[14]}$

The risk of VTE is increased 5 - 10-fold in pregnancy. The hypercoagulability of pregnancy persists for several weeks after delivery, where the greatest risk for VTE is in the early postpartum period. The recent decline in maternal deaths from VTE can be attributed to the use of thromboprophylaxis in high-risk women. ${ }^{[15,16]}$

VTE risk assessment is recommended early during pregnancy and in the postpartum period. Risk factors include previous VTE, family history of VTE, hereditary thrombophilia, antiphospholipid syndrome (APS), medical comorbidities, significant pregnancy complications, caesarean delivery, prolonged antepartum immobilisation and clinical risk factors, such as increased body mass index (BMI), age $>35$ years and parity $\geq 3$. High-risk patients should be managed in conjunction with a haematologist and, in the case of APS, a rheumatologist. Antepartum and postpartum thromboprophylaxis with low-molecular-weight heparin (LMWH) and low-dose aspirin is recommended in women with APS and previous VTE. Higher doses of LMWH may be required. ${ }^{[16]}$

The ideal anticoagulant in pregnancy does not cross the placenta and can be easily reversed. The oral direct thrombin and FXa inhibitors should not be used in pregnancy, as the molecules are small and cross the placenta. Warfarin is associated with a teratogenic effect, especially between 6 and 12 weeks' gestation. Furthermore, there is an increased risk of miscarriage, prematurity and fetal bleeding (including intracranial haemorrhage resulting in brain damage) at any time during pregnancy. LMWH is the preferred anticoagulant. Antepartum prophylaxis should be initiated early in 
pregnancy. Postpartum thromboprophylaxis should be continued for 6 weeks in high-risk women, for 10 days in intermediate-risk women and at least until discharge from hospital in low-risk women. There is an increased dose requirement for LMWH during pregnancy because of increased volume of distribution and renal clearance. Regular anti-Xa monitoring is therefore recommended.

\section{Obesity}

Obesity is defined as a BMI $\geq 30 \mathrm{~kg} / \mathrm{m}^{2}$. Owing to its increasing prevalence, obesity has emerged as an important risk factor for VTE in addition to myocardial infarction and stroke in high-income countries. In South Africa (SA), a resource-limited country, there is a bimodal distribution, with a rise in obesity, particularly in urban settings. Central obesity is characterised by chronic inflammation and impaired fibrinolysis, which are the two major pathways that contribute to VTE risk. ${ }^{[17]}$

As obesity is considered a moderate risk factor for VTE in highrisk circumstances, such as hospitalised medical patients, surgery and immobilisation, thromboprophylaxis should be considered. Higher doses of LMWH are often required for the prevention of VTE in obese patients. ${ }^{[18]}$ Laboratory monitoring of LMWH therapy in obese patients using anti-Xa levels is indicated. There are limited clinical data available on the use of direct oral anticoagulants (DOACs), such as dabigatran, rivaroxaban and apixaban, for patients with a $\mathrm{BMI} \geq 40 \mathrm{~kg} / \mathrm{m}^{2}$. DOAC activity levels should be monitored in these patients using a drug-specific peak level. ${ }^{[19]}$

\section{Cancer}

The risk of VTE is significantly increased among patients with cancer, with an estimated relative risk 4 - 7-fold above that of the background population. ${ }^{[20]}$ The basis for malignancy-associated VTE include factors related to the cancer itself (such as expression of procoagulant molecules, particularly TF, by the tumour cells), chemotherapy-related endothelial injury and/or increased frequency of other risks for VTE (e.g. major surgery, immobilisation, vascular obstruction by tumour masses or indwelling venous catheters). ${ }^{[21]}$ VTE rates are particularly high in certain types of cancer (such as haematological neoplasms, and tumours of the stomach, pancreas, brain, lung and breast), ${ }^{[21,22]}$ in advanced-stage disease and when therapeutic agents with increased thrombogenic potential are used, such as platinum-based chemotherapy agents, immunomodulatory drugs (e.g. thalidamide, tamoxifen) and haemopoietic-stimulating agents (e.g. erythropoietin). ${ }^{[21,22]}$

Despite the increased risk of VTE in association with cancer, routine primary prophylaxis among ambulatory individuals with a malignancy is not advocated, as the rate of bleeding complications is also elevated with LMWH-based VTE prophylaxis. ${ }^{[21,22]}$ However, thromboprophylaxis is recommended for hospital inpatients, particularly following major surgery, such as abdomenopelvic surgery, which necessitates prophylaxis with LMWH for 30 days. ${ }^{[21]}$ Among patients with confirmed thrombosis, therapeutic doses of LMWH have shown superior efficacy compared with oral vitamin K antagonists, possibly due to difficulties in achieving predictable anticoagulation in patients with cancer owing to numerous drug-drug interactions, poor diet/vomiting (which can affect vitamin K bio-availability) or associated liver/renal impairment. ${ }^{[21]}$ Early indications suggest that the DOACs, rivaroxaban, apixaban and edoxaban (of which only rivaroxaban is currently available in SA), may be acceptable alternatives for the management of VTE, but caution is warranted if potent inducers or inhibitors of CYP450 are used concomitantly. ${ }^{[21,23]}$
Furthermore, these agents have been associated with an increased risk of bleeding in patients with gastrointestinal cancers, and should be used with caution in this group. ${ }^{[23]}$ This has led the Scientific and Standardization Committee on Haemostasis and Malignancy of the International Society of Thrombosis and Hemostasis (ISTH) to recommend the use of DOACs only in patients with a low bleeding risk who do not use agents likely to cause drug-drug interactions. ${ }^{[24]}$ Cancerassociated thrombosis should be treated for at least $3-6$ months, and as the risk of recurrence is increased, ongoing anticoagulation should be considered while the patient has active cancer. ${ }^{[23]}$

Among otherwise apparently well patients who present with unprovoked thrombosis, a small proportion can be demonstrated to be harbouring occult malignancies. Limited cancer screening is therefore recommended by the ISTH in this group of patients, including a clinical evaluation, chest radiograph, full blood count, liver function testing, serum calcium, urinalysis and ageappropriate screening tests (e.g. prostate-specific antigen level, mammography). ${ }^{[25]}$ Patients who experience recurrent VTE while on anticoagulant therapy or bilateral deep-vein thrombosis are at particularly high risk of occult cancer, and may benefit from a more extensive work-up in the case of underlying malignancy. ${ }^{[20]}$

\section{Sickle cell disease}

VTE is a common comorbid condition found in sickle cell disease (SCD) and is associated with increased mortality.

The incidence of thrombotic stroke and pulmonary embolism is higher than in persons without SCD. ${ }^{[2,27]}$ SCD is also a risk factor for pregnancy-related VTE (odds ratio (OR) 20.3). ${ }^{[28]}$ As the pathogenesis of vaso-occlusion is multifactorial, the precise role of hypercoagulability is not certain. The preponderance of evidence demonstrates increased platelet and coagulation activation. Prothrombin fragment 1.2, a marker of thrombin generation, is elevated in SCD. Phosphatidyl serine exposure on the surface of the red cell membrane is thought to serve as a trigger, with contributing factors, such as functional asplenia and nitric oxide depletion due to chronic haemolysis. ${ }^{[29]}$

Prophylactic anticoagulation is recommended during potentially triggering events such as perioperative periods and indwelling venous catheters. Meaningful data on the long-term use of anticoagulants or antiplatelet agents are lacking owing to small and/or poorly controlled studies.

\section{Clinical entities characterised by a thrombotic tendency The antiphospholipid syndrome}

APS is associated with a predilection to thrombosis and recurrent morbidity related to pregnancy. Thrombosis can affect any vessel (arterial, venous or the microvasculature), and recurs often. The pathogenesis of thrombotic APS is not well understood, but the binding of autoantibodies directed against various phospholipids or phospholipid-binding proteins (including cardiolipin and beta-2 glycoprotein I) results in: (i) upregulated monocyte and endothelial cell TF expression; (ii) platelet activation; and (iii) disruption of natural anticoagulant mechanisms (such as the activated PC pathway and the annexin $\mathrm{V}$ shield (which blocks access of coagulation factors to procoagulant phospholipid surfaces). ${ }^{[30]}$ The most common thrombotic manifestations include cerebrovascular accidents, lowerlimb deep-vein thrombosis and pulmonary embolism. ${ }^{[3]}$ APS should be suspected if:

- thrombosis occurs in a young patient, at an unusual site or recurs ${ }^{[31]}$ 
- other clinical features associated with APS are present, including underlying systemic lupus erythematosus, mild thrombocytopenia, autoimmune haemolysis, livedo reticularis, cardiac valve thickening or vegetations, thrombotic microangiopathy, recurrent miscarriages, nephropathy, neurological abnormalities or unexplained prolongation of the activated partial thromboplastin time (aPTT) ${ }^{[31]}$

The diagnosis is based on the revised Sapporo criteria, which require demonstration of $\geq 1$ laboratory criteria with $\geq 1$ clinical manifestations (Table 2). ${ }^{[32]}$ The laboratory findings (i.e. anticardiolipin antibodies, anti-beta-2 glycoprotein antibodies or a lupus anticoagulant) are somewhat nonspecific, and can occur transiently after a viral infection or secondary to underlying autoimmune pathology, malignancy or drug exposure. Persistence of these abnormalities must therefore be demonstrated for $>12$ weeks with appropriate clinical manifestations before a diagnosis of APS can be made.

Management of thrombotic APS entails lifelong anticoagulation with a vitamin $\mathrm{K}$ antagonist (such as warfarin and a target international normalised ratio (INR) of 2 - 3), with optimisation of other risk factors for thrombosis (e.g. hypertension, hypercholesterolaemia). Concomitant use of aspirin is advocated for arterial thrombosis in patients with significant risk factors for cardiovascular disease. In the event of recurrent thrombosis despite adequate anticoagulation (a well-described complication), options include the addition of aspirin, use of high-dose vitamin $\mathrm{K}$ antagonist therapy (target INR 3 - 4) and/or an alternative anticoagulant (particularly LMWH). Currently, DOACs are not advocated for the management of APS, as their efficacy and safety are not proven. ${ }^{[31]}$

\section{Heparin-induced thrombocytopenia}

Heparin-induced thrombocytopenia (HIT) is an immunological complication of heparin therapy that is associated with a decrease in the platelet count (usually resulting in thrombocytopenia) and an increase in the risk of thrombosis (both venous and arterial). ${ }^{[33,34]}$ It typically develops 5 - 10 days after the commencement of heparin therapy, except in patients with a history of recent heparin exposure, in whom the clinical features may manifest more rapidly. It is caused by the production of antibodies directed against a complex of heparin and platelet FIV (PF4), which is an endogenous platelet protein released from the alpha granules of platelets on platelet activation. ${ }^{[3,34]}$ When bound to heparin-PF4 complexes, these antibodies cross-link FcYRIIa receptors on platelets and monocytes, which generates a prothrombotic state by triggering platelet activation and $\mathrm{TF}$ expression on the surface of monocytes. ${ }^{[34]}$

These antibodies occur fairly commonly in patients exposed to heparin, but HIT occurs in only $0.2-3.0 \%$ of such patients. ${ }^{[34]}$ Factors associated with a high risk of HIT include a history of recent major surgery, unfractionated heparin (UFH) exposure and use of therapeutic doses of LMWH. ${ }^{[34]}$ Notably, the risk of HIT is significantly lower with prophylactic doses of LMWH. ${ }^{[33,34]}$ Diagnosis is primarily based on clinical suspicion, with the presence of thrombocytopenia or thrombosis at the appropriate time interval following heparin exposure without an alternative cause (assessed in the $4 \mathrm{~T}$ score) (Table 3). HIT can be excluded if the $4 \mathrm{~T}$ score is low $(<4)$, but requires laboratory confirmation when the score is intermediate or high. ${ }^{[33,34]}$ Laboratory tests available in SA include functional tests that detect platelet activation upon heparin exposure, as well as immunoassays that demonstrate antiheparin/PF4 antibodies. The immunoassays have superior sensitivity, but poorer specificity; therefore, while a negative result excludes a diagnosis of HIT, a positive result is only confirmatory when the $4 \mathrm{~T}$ score is high. ${ }^{[34]}$ In contrast, the sensitivity of the functional assay is lower, but its specificity is higher, and a positive functional test result therefore confirms a diagnosis of HIT, even in patients with an intermediate $4 \mathrm{~T}$ score ${ }^{[34]}$ Knowledge regarding the type of assay performed is important; therefore, consultation with regional laboratory services is necessary.

Management of HIT entails immediate cessation of heparin therapy and commencement of an agent that does not cross-react with the antiheparin/PF4 antibodies. Importantly, the results of laboratory assays should not be awaited before initiating the alternative anticoagulant, as the risk of thrombosis is very high $(\sim 50 \%),{ }^{[33]}$ and the test results are often not available for a few days. Agents that can be employed include parenteral inhibitors of thrombin (argatroban), FXa (fondaparinux) or the DOACs (dabigatran or rivaroxiban). ${ }^{[35]}$ LMWH should be avoided. Warfarin is contraindicated, as warfarinrelated PC deficiency may cause significant extension of the thrombus, with a risk of venous gangrene. ${ }^{[33,34]}$ In the absence

Table 2. Revised Sapporo classification criteria for the diagnosis of antiphospholipid syndrome ${ }^{[32]}$

\section{Clinical criteria}

Objectively confirmed arterial, venous or small-vessel thrombosis in any organ

Pregnancy morbidity

- $\geq 1$ unexplained death/s of a morphologically normal fetus at or beyond the 10th week of gestation

- $\geq 1$ premature birth/s of a morphologically normal neonate before the 34 th week of gestation due to eclampsia, severe pre-eclampsia or placental insufficiency

- $\geq 3$ unexplained, consecutive spontaneous miscarriages before the 10th week of gestation (without maternal anatomical or paternal and maternal chromosomal abnormalities)

Laboratory criteria

LA on $\geq 2$ occasions at least 12 weeks apart (detected by prolongation of a phospholipid-dependent clotting time, e.g. DRVVT, lupus-sensitive aPTT)

ACL antibody (IgG and/or IgM) measured by an ELISA-based immunological assay, present in medium- or high-titre (i.e. $\geq 40$ GPL or MPL), on $\geq 2$ occasions, at least 12 weeks apart

Anti- $\beta_{2}$-glycoprotein I (IgG and/or IgM) measured by an ELISA-based immunological assay, present in medium- or high-titre (i.e. $\geq 99$ th percentile), on $\geq 2$ occasions, at least 12 weeks apart

Patients with LA positivity are at greater risk of clinical events, particularly those with triple positivity for LA, ACL and anti- $\beta_{2}$-glycoprotein I $\mathrm{LA}=$ lupus anticoagulant; DRVVT = dilute Russell viper venom time; $\mathrm{aPTT}=$ activated partial thromboplastin time; $\mathrm{ACL}$ = anticardiolipin; ELISA = enzyme-linked immunosorbent assay; $\mathrm{GPL}=\mathrm{IgG}$ phospholipid; MPL = IgM phospholipid. 
Table 3. 4T scoring system ${ }^{\star}$ for the diagnosis of heparin-induced thrombocytopenia

\begin{tabular}{|c|c|c|c|}
\hline & +2 & +1 & $\mathbf{0}$ \\
\hline Thrombocytopenia & $\begin{array}{l}\text { Platelet count fall }>50 \% \text { and } \\
\text { platelet nadir } \geq 20\end{array}$ & $\begin{array}{l}\text { Platelet count fall } 30-50 \% \text { or } \\
\text { platelet nadir } 10-19\end{array}$ & $\begin{array}{l}\text { Platelet count fall }<30 \% \text { or } \\
\text { platelet nadir }<10\end{array}$ \\
\hline Timing & $\begin{array}{l}\text { Definite onset between days } 5 \text { and } \\
10 \text { or platelet count fall } \leq 1 \text { day } \\
\text { if heparin exposure in the past } \\
30 \text { days }\end{array}$ & $\begin{array}{l}\text { Probable onset between } \\
\text { days } 5 \text { and } 10 \text {, but not clear; } \\
\text { onset after day } 10 \text { or platelet } \\
\text { count fall } \leq 1 \text { day if heparin } \\
\text { exposure in the past } 30 \text { - } 100 \text { days }\end{array}$ & $\begin{array}{l}\text { Platelet count fall }<4 \text { days } \\
\text { without recent exposure }\end{array}$ \\
\hline Thrombosis & $\begin{array}{l}\text { New thrombosis or skin necrosis/ } \\
\text { acute systemic reaction after } \\
\text { intravenous heparin bolus }\end{array}$ & $\begin{array}{l}\text { Progressive or recurrent } \\
\text { thrombosis; suspected } \\
\text { thrombosis (not proven) }\end{array}$ & None \\
\hline Other causes for thrombocytopenia & None apparent & Possible & Definite \\
\hline
\end{tabular}

of thrombosis, alternative anticoagulation treatment should be maintained for 4 weeks. If thrombosis is present, warfarin should be commenced once the platelet count has normalised ( $>150 \times 10^{9} / \mathrm{L}$ on $\geq 2$ occasions), and continued for 3 months. ${ }^{[34]}$

\section{Paroxysmal nocturnal haemoglobinuria}

Paroxysmal nocturnal haemoglobinuria $(\mathrm{PNH})$ is a rare clonal disorder that is caused by an acquired mutation of the PIG-A gene, which encodes an anchor molecule (glycosylphosphatidylinositol (GPI)) required for affixing a number of proteins on cell surfaces (including the complement-inactivating proteins CD55 and CD59). ${ }^{[36]}$ Cells with GPI deficiency are thus vulnerable to complement activation on their surfaces, which can cause cell lysis (in the case of red cells), cellular activation (in the case of platelets) and indirectly, endothelial dysfunction. ${ }^{[3,37]}$ This accounts for the clinical manifestations of $\mathrm{PNH}$, which include episodic haemoglobinuria (due to intravascular haemolysis) and a predilection to thrombosis. $\mathrm{PNH}-$ positive clones may also be seen in patients with underlying haematological disorders (particularly aplastic anaemia), although typical clinical manifestations of $\mathrm{PNH}$ are unusual in this setting. PNH should be suspected in patients with thrombosis, who also have cytopenias, iron deficiency (as a consequence of long-standing haemoglobinuria), evidence of intravascular haemolysis or when thrombosis is detected at unusual sites (particularly intra-abdominal or cerebral veins). ${ }^{[37]}$ Diagnosis is made on flow cytometry, which is used to detect cell populations that do not express GPI-linked surface antigens. ${ }^{[36]} \mathrm{PNH}$-associated thrombosis can be very difficult to manage; these patients should be referred to specialist haematology centres.

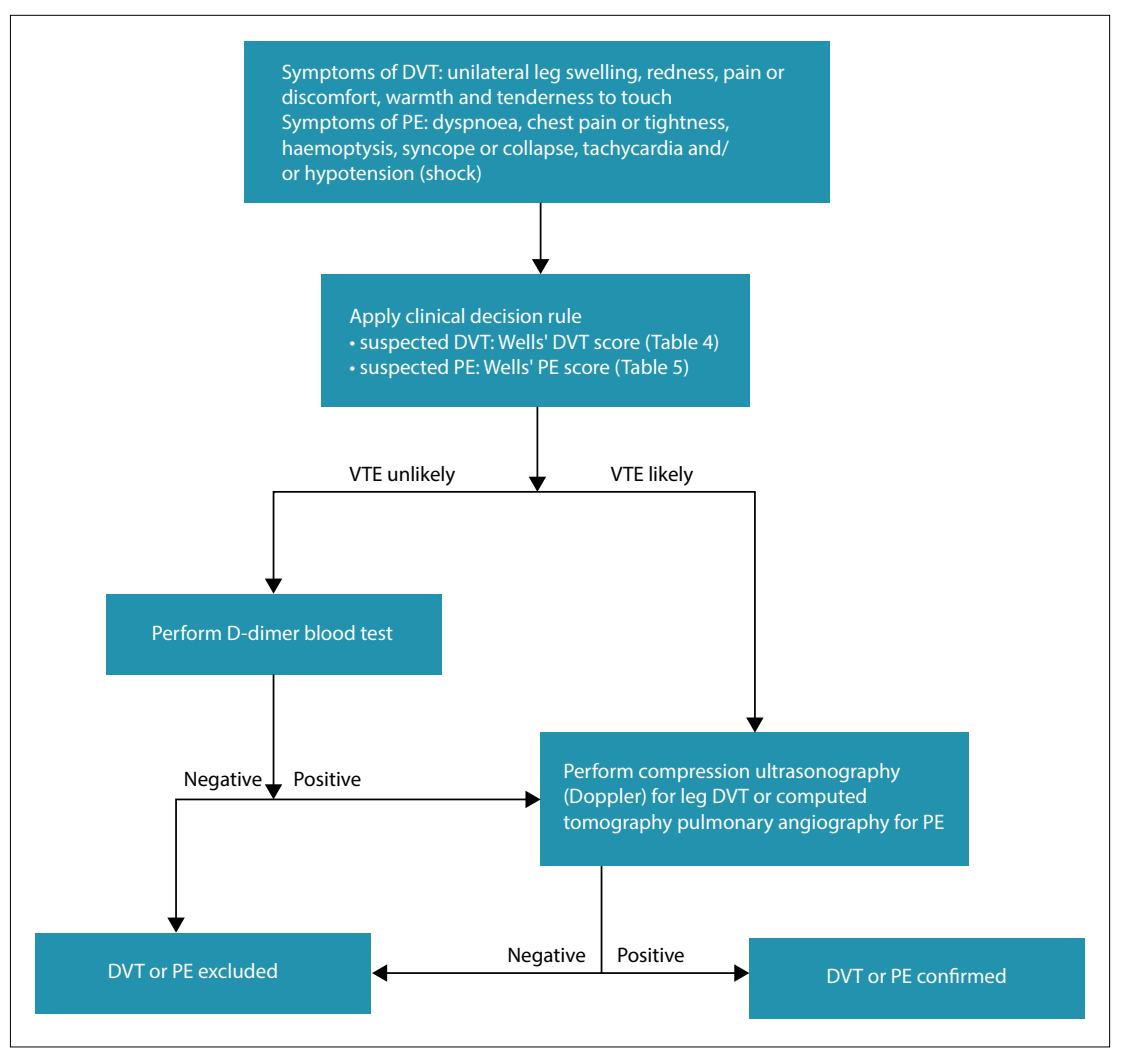

Fig. 2. Diagnostic approach for patients with suspected venous thromboembolism. (VTE = venous thromboembolism; $D V T=$ deep-vein thrombosis; $P E=$ pulmonary embolism.)

\section{Diagnostic considerations}

The algorithm in Fig. 2 outlines a diagnostic approach for suspected thromboembolic disease. ${ }^{[38]}$ Wells' scores for clinical assessment of deep-vein thrombosis and pulmonary embolism appear in Tables 4 and 5, respectively.

\section{Thrombophilia testing}

FV Leiden (which does not occur among black population affinity groups) and prothrombin gene (20210A) mutations together with antiphospholipid antibodies constitute the most prevalent thrombophilias. Deficiencies of the natural anticoagulants (PC, PS and antithrombin) and elevated homocysteine levels tend to be of low yield, and testing for these should generally be limited to patients with particularly high-risk and unique presentations.

Thrombophilia testing need not be done in patients with a clear provoking event, such as recent major orthopaedic surgery in the absence of other factors suspicious for thrombophilia, or in those with underlying malignancy, as results are unlikely to alter the treatment plan.

Samples for FV Leiden and prothrombin gene (20210A) mutations, as well as anti- 


\section{Table 4. Wells' score ${ }^{\star}$ for deep-vein thrombosis}

\begin{tabular}{|c|c|}
\hline Criteria & Points \\
\hline Active cancer (patient either received treatment during the previous 6 months or currently receiving palliative treatment) & +1 \\
\hline Paralysis, paresis or recent cast immobilisation of the lower extremities & +1 \\
\hline Recently bedridden for $\geq 3$ days, or major surgery within the previous 12 weeks, requiring general or regional anaesthesia & +1 \\
\hline Localised tenderness along the distribution of the deep-venous system of the leg & +1 \\
\hline Swelling of entire leg & +1 \\
\hline Calf swelling at least $3 \mathrm{~cm}$ larger than that on the asymptomatic side (measured $10 \mathrm{~cm}$ below tibial tuberosity) & +1 \\
\hline Pitting oedema confined to the symptomatic leg & +1 \\
\hline Collateral superficial veins (non-varicose) & +1 \\
\hline Previously documented DVT & +1 \\
\hline Alternative diagnosis at least as likely as DVT & -2 \\
\hline
\end{tabular}

\section{Table 5. Wells' score* for pulmonary embolism}

\begin{tabular}{ll}
\hline Criteria & Points \\
\hline Clinical signs and symptoms of DVT & +3 \\
PE is the most likely diagnosis or equally likely v. & +3 \\
another diagnosis & \\
Heart rate $>100 /$ min & +1.5 \\
Immobilisation for at least 3 days or surgery in the & +1.5 \\
previous 4 weeks & \\
Previous, objectively diagnosed PE or DVT & +1.5 \\
Haemoptysis & +1 \\
Malignancy with treatment within 6 months or & +1 \\
palliation & \\
$\begin{array}{l}\text { DVT }=\text { deep-vein thrombosis; } P E=\text { pulmonary embolism. } \\
{ }^{\prime}>6=\text { high probability; } 2-6=\text { moderate probability; }<2=\text { low risk. }\end{array}$ &
\end{tabular}

phospholipid antibodies (excluding functional lupus anticoagulant), can be collected in the acute phase of thrombosis, as these results are not affected by acute thrombosis or anticoagulants. Functional lupus anticoagulant testing, as well as testing for PC and PS and antithrombin deficiencies, should preferably be delayed until completion of anticoagulation therapy. However, these can be collected in the acute phase prior to initiation of therapy in those patients who might require lifelong anticoagulation.

\section{Conclusions}

The aetiology and pathogenesis of acquired thromboses are fairly diverse and depend to a large extent on the primary pathology and associated compounding factors.

An accurate history (with knowledge of pre-existing conditions), physical examination and appropriate investigations are imperative for proper diagnosis and management of acquired thrombophilia

\section{Declaration. None}

\section{Acknowledgements. None.}

Author contributions. NAA: concept, design and content; JV: design and content; SL: content; ES: content; and BJ: content.

Funding. None.

Conflicts of interest. None.

1. Alli NA, Vaughan J, Louw S, Schapkaitz E, Jacobson B. Thrombotic disorders (part 1). S Afr Med 2020;110(2):83-87. https://doi.org/10.7196/SAMJ.2020.v110i2.14594

2. Gale AJ. Current understanding of hemostasis. Toxicol Pathol 2011;39(1):273-280. https://doi. org/10.1177/0192623310389474
3. Sud R, Khorana AA. Cancer-associated thrombosis: Risk factors, candidate biomarkers and a risk model. Thromb Res 2009;123(suppl 4):S18-S21. https://doi.org/10.1016/S0049-3848(09)70137-9

4. Bode M, Mackman N. Regulation of tissue factor gene expression in monocytes and endothelial cells: Thromboxane A2 as a new player. Vascul Pharmacol 2014;62(2):57-62. https://doi.org/10.1016/j. vph.2014.05.005

5. Dentan C, Epaulard O, Seynaeve D, Genty C, Bosson JL. Active tuberculosis and venous thromboembolism: Association according to international classification of diseases. Clin Infect Dis 2014;58(4):495-501. https://doi.org/10.1093/cid/cit780

6. Hodkinson KE, Mahlangu JN. Deep-vein thrombosis in the era of high HIV and tuberculosis prevalence: A prospective review of its diagnosis and treatment in a quaternary centre. S Afr Med J 2017:107(10):859-863. https://doi. org/10.7196/SAMJ2017 v107i10.12443

7. Jong E, Louw S, Meijers JC, et al. The hemostatic balance in HIV-infected patients with and without antiretroviral therapy: Partial restoration with antiretroviral therapy. AIDS Patient Care STDS 2009;23(12):1001-1007. https://doi.org/10.1089/apc.2009.0173

8. Mayne ES, Louw S. Good fences make good neighbors: Human immunodeficiency virus and vascular disease. Open Forum Infect Dis 2019;6(11):ofz303. https://doi.org/10.1093/ofid/ofz303

9. Jacobson BF, Louw S, Buller H, et al. Venous thromboembolism: Prophylactic and therapeutic practice guideline. S Afr Med J 2013;103(4):260-267. https://doi.org/10.7196/samj.6706

10. Dager WE, Tsu LV, Pon TK. Considerations for systemic anticoagulation in ESRD. Semin Dial 2015;28(4):354-362. https://doi.org/10.1111/sdi.12376

11. Lutz J, Menke J, Sollinger D, Schinzel H, Thurmel K. Haemostasis in chronic kidney disease. Nephrol Dial Transplant 2014;29(1):29-40. https://doi.org/10.1093/ndt/gft209

12. Giannotta M, Tapete G, Emmi G, Silvestri E, Milla M. Thrombosis in inflammatory bowel diseases: What's the link? Thromb J 2015;13:14. https://doi.org/10.1186/s12959-015-0044-2

13. Zezos P, Kouklakis G, Saibil F. Inflammatory bowel disease and thromboembolism. World J Gastroenterol 2014;20(38):13863-13878. https://doi.org/10.3748/wig.v20.i38.13863

14. Stegeman BH, de Bastos M, Rosendaal FR, et al. Different combined oral contraceptives and the risk of venous thrombosis: Systematic review and network meta-analysis. BMJ 2013;347:f5298. https://doi. org $/ 10.1136 / \mathrm{bmj} . .5298$

15. Wilkinson $\mathrm{H}$, Trustees, Medical Advisers. Saving mothers' lives. Reviewing maternal deaths to make motherhood safer: 2006 - 2008. Br J Obstet Gynaecol 2011;118(11):1402-1404. https://doi. org/10.1111/j.1471-0528.2011.03097.x

16. Bates SM, Greer IA, Middeldorp S, et al. VTE, thrombophilia, antithrombotic therapy, and pregnancy: Antithrombotic therapy and prevention of thrombosis. Chest 2012;141(2 Suppl):e691S-e736S. https:// doi.org/10.1378/chest.11-2300

17. Morange PE, Alessi MC. Thrombosis in central obesity and metabolic syndrome: Mechanisms and epidemiology. Thromb Haemost 2013;110(4):669-680. https://doi.org/10.1160/TH13-01-0075

18. Frederiksen SG, Hedenbro JL, Norgren L. Enoxaparin effect depends on body-weight and current doses may be inadequate in obese patients. Br J Surg 2003;90(9):1165-1166.

19. Martin K, Beyer-Westendorf J, Davidson BL, et al. Use of the direct oral anticoagulants in obese patients: Guidance from the SSC of the ISTH. J Thromb Haemost 2016;14(6):1308-1313. https://doi. org/10.1111/jth.13323

20. Timp JF, Braekkan SK, Versteeg HH, Cannegieter SC. Epidemiology of cancer-associated venous thrombosis. Blood 2013:122(10):1712-1723 https.// doi org/10.1182/blood-2013-04-460121

21. Fernandes C,, Morinaga LTK, Alves JL, jun., et al. Cancer-associated thrombosis: The when, how and why. Eur Respir Rev 2019;28(151):piil80119. https://doi.org/10.1183/16000617.0119-2018

22. Streiff MB. Thrombosis in the setting of cancer. Hematol Am Soc Hematol Educ Program 2016;2016(1):196-205. https://doi.org/10.1182/asheducation-2016.1.196

23. Kraaijpoel N, Carrier M. How I treat cancer-associated venous thromboembolism. Blood 2019;133(4):291-298. https://doi.org/10.1182/blood-2018-08-835595

24. Khorana AA, Noble S, Lee AYY, et al. Role of direct oral anticoagulants in the treatment of cancer associated venous thromboembolism: Guidance from the SSC of the ISTH. J Thromb Haemost 2018;16(9):1891-1894. https://doi.org/10.1111/jth.14219

25. Delluc A, Antic D, Lecumberri R, Ay C, Meyer G, Carrier M. Occult cancer screening in patients with venous thromboembolism: Guidance from the SSC of the ISTH. J Thromb Haemost 2017;15(10):2076-2079. https://doi.org/10.1111/jth.13791

26. Liffering WM, Brouwer JL, Veeger NJ, et al. Selective testing for thrombophilia in patients with first venous thrombosis: Results from a retrospective family cohort study on absolute thrombotic risk for currently known thrombophilic defects in 2479 relatives. Blood 2009;113(21):5314-5322. https://doi. org/10.1182/blood-2008-10-184879

27. Spencer FA, Emery C, Joffe SW, et al. Incidence rates, clinical profile, and outcomes of patients with venous thromboembolism. The Worcester VTE study. J Thromb Thrombolysis 2009;28(4):401-409. https://doi.org/10.1007/s11239-009-0378-3

28. Noubiap JJ, Temgoua MN, Tankeu R. Sickle cell disease, sickle trait and the risk for venous thromboembolism: A systematic review and meta-analysis. Thromb J 2018;16:27. https://doi.org/10.1186/s12959-018-0179-z

29. De Franceschi L, Cappellini MD, Olivieri O. Thrombosis and sickle cell desease. Semin Thromb Hemost 2011;37(3):226-236. https://doi.org/10.1055/s-0031-1273087

30. Linnemann B. Antiphospholipid syndrome - an update. Vasa 2018;47(6):451-464. https://doi. org/10.1024/0301-1526/a000723

31. Garcia D, Erkan D. Diagnosis and management of the antiphospholipid syndrome. N Engl J Med 2018;379(13):1290. https://doi.org/10.1056/NEJMc1808253 
32. Miyakis S, Lockshin MD, Atsumi T, et al. International consensus statement on an update of the classification criteria for definite antiphospholipid syndrome (APS). J Thromb Haemost 2006;4(2):295-306. https://doi.org/10.1111/j.1538-7836.2006.01753.x

33. Greinacher A. Heparin-induced thrombocytopenia. N Engl J Med 2015;373(19):1883-1884

34. Arepally GM. Heparin-induced thrombocytopenia. Blood 2017;129(21):2864-2872. https://doi. org/10.1056/NEJMc1510993

35. Cuker A, Arepally GM, Chong BH, et al. American Society of Hematology 2018 guidelines for management of venous thromboembolism: Heparin-induced thrombocytopenia. Blood Adv
mation management of venous thromboembolism: Heparin-induced throm
2018;2(22):3360-3392. https://doi.org/10.1182/bloodadvances.2018024489
36. Luzzatto L. Recent advances in the pathogenesis and treatment of paroxysmal nocturnal hemoglobinuria. F1000Res 2016;5:pii. https://doi.org/10.12688/f1000research.7288.1

37. Hill A, Kelly RJ, Hillmen P. Thrombosis in paroxysmal nocturnal hemoglobinuria. Blood 2013;121(25):4985-4996,5105. https://doi.org/10.1182/blood-2012-09-311381

38. Tritschler T, Kraaijpoel N, le Gal G, Wells PS. Venous thromboembolism: Advances in diagnosis and treatment. JAMA 2018;320(15):1583-1594. https://doi.org/10.1001/jama.2018.14346

Accepted 31 January 2020. 